\title{
Budget Impact Analysis Highlighting the Cost-Saving Potential of Generic Tenofovir Disoproxil Based Antiretroviral Therapy in Comparison with Tenofovir Alafenamide Based Treatment Regimens in the German Healthcare System
}

Matthias Stoll', Phoebe Balkin², Joachim Goldbach ${ }^{3}$, Philipp Weber ${ }^{4}$, Baerbel Peetroons ${ }^{5}$ and Sameer B Gokhale ${ }^{6 *}$

${ }^{1}$ Clinic for Immunology and Rheumatology, Unit for Infectious Diseases, Medical University of Hanover (MHH), Carl Neuberg Hanover, Germany ${ }^{2} \mathrm{Health}$ Economics Modeling Project, Product Lifecycle Services, Novartis Business Services Dublin, Elm Park, Dublin, Ireland

${ }^{3}$ HIV Department, Muensterstr, Mainz, Germany

${ }^{4}$ Market Access Department, Hexal, Industriestraße Holzkirchen, Germany

${ }^{5}$ Medical Department, Hexal AG, Industriestraße, Holzkirchen, Germany

${ }^{6}$ Patient Access Services, Novartis Global Service Center, Hyderabad, Telangana, India

\begin{abstract}
Objective: The fixed dose combination (FDC) of tenofovir disoproxil (TD) fumarate/emtricitabine (FTC) has been the most widely used backbone treatment for HIV until recently. In 2016, tenofovir alafenamide (TAF)/FTC became available as FDC, which promises superior renal and bone safety. However, in November 2016, the German Federal Joint Committee concluded that there is no additional benefit to the use of TAF versus TD fumarate. The decision of choosing TD or TAF in FDC with FTC is of economic impact as cost-saving generics (Gx-TD/FTC) became available in Germany in August 2017.
\end{abstract}

Methods: A budget impact analysis (BIA) was conducted to estimate the impact of Gx-TD/FTC on the German healthcare system for 5-years. The BIA compared a theoretical setting with and without introduction of Gx-TD/FTC; calculating the respective costs for antiretroviral treatment, monitoring, and adverse event management.

Results: Based on the model assumptions, low and high values of year-wise savings with Gx-TD/FTC throughout 5 years were estimated. The scenario of Gx-TD/FTC use growing to $35 \%$ by year 5 , deemed the most realistic scenario(switch from TDF/FTC to Gx-TD/FTC only), would result in a saving of $€ 22.6$ million in first year and a maximum saving of $€ 31.8$ million by second year. In a maximum patient share scenario (80\%) for Gx-TD/FTC which includes switch from branded TD fumarate/FTC and TAF/FTC, savings increased from €22.6 million annually to a maximum of $€ 62.1$ million.

Conclusion: The estimated savings are based on a conservative approach and will be higher with increasing generic price rebates. Additionally, this analysis makes cautious assumptions in favor of patient safety and care pathways. This analysis highlights the potential for substantial and sustainable cost savings by the use of Gx-TD/FTC for the German healthcare system which is most pronounced when patients from branded TD fumarate/FTC and TAF/ FTC are switched to this generic treatment.

Keywords: Budget impact analysis; Cost saving; Antiretroviral treatment; Tenofovir disoproxil fumarate; Tenofovir alafenamide; German healthcare system; Generics; Scenario analysis; Economic modeling

\section{Introduction}

Combined antiretroviral (ARV) therapy (cART) has been the gold standard for the treatment of human immunodeficiency virus (HIV)infection since the midst of the 1990s. By early initiation of cART an almost normalized life expectancy of those infected with HIV could be achieved by the use of currently recommended treatment regimens [1]. By significantly reducing HIV-associated morbidity and mortality, a sustainable reduction of costs could be achieved. As a result however, cART has become the dominant cost factor and has increased up to $85 \%$ of direct costs of disease for HIV infection in Europe [2-5].

In order to control pharmaceutical prices for patented drugs and to curb increasing pharmaceutical spending, important regulations have been introduced by the German legislator in recent years. First, the German law to strengthen competition in the health-care sector since 2007 promotes the spread of generic drugs after patent expiration and enhances the number of discount agreements between drug manufacturers and health insurance companies [6]. Second, the Act for Restructuring the Pharmaceutical Market in Statutory
Health Insurance (AMNOG) since 2011 mandates pharmaceutical manufacturers to demonstrate a therapeutic benefit for innovative pharmaceutical agents. The result of price negotiations between the Federal Association of Statutory Health Insurance Funds and the pharmaceutical manufacturer depends on the level of additional benefit of the pharmaceutical product. The extent of additional benefit is assessed in a complex process, based on an external expertise about a

*Corresponding author: Sameer B Gokhale, Novartis Global Service Center, Salarpuria-Sattva Knowledge City, Inorbit Mall Road, Raidurgam, Rangareddy District, Hyderabad, Telangana, 500 032, India, Tel: +91-9004471299; E-mail sameer.gokhale@novartis.com

Received December 21, 2017; Accepted January 10, 2018; Published January 17,2018

Citation: Stoll M, Balkin P, Goldbach J, Weber P, Peetroons B, et al. (2018) Budge Impact Analysis Highlighting the Cost-Saving Potential of Generic Tenofovir Disoproxil Based Antiretroviral Therapy in Comparison with Tenofovir Alafenamide Based Treatment Regimens in the German Healthcare System. J AIDS Clin Res 9: 754. doi: 10.4172/2155-6113.1000754

Copyright: (C) 2018 Stoll M, et al. This is an open-access article distributed unde the terms of the Creative Commons Attribution License, which permits unrestricted use, distribution, and reproduction in any medium, provided the original author and source are credited. 
Citation: Stoll M, Balkin P, Goldbach J, Weber P, Peetroons B, et al. (2018) Budget Impact Analysis Highlighting the Cost-Saving Potential of Generic Tenofovir Disoproxil Based Antiretroviral Therapy in Comparison with Tenofovir Alafenamide Based Treatment Regimens in the German Healthcare System. J AIDS Clin Res 9: 754. doi: 10.4172/2155-6113.1000754

Page 2 of 9

structured dossier to be provided by the manufacturer [7]. As a result, prices for newly approved antiviral medicines for the treatment of HIV or hepatitis $\mathrm{C}$ virus (HCV) infection in Germany have since declined compared with that in other industrialized nations [8].

By contrast, the price pressure from generic ARVs, which became available in the German market since 2013, was relatively low. A majority of initially availablegeneric drugs, i.e., zidovudine, nevirapine and efavirenz are no longer recommended as a preferred option in current national [9] and international [10,11] treatment guidelines. Their market share has therefore declined and budget impact has remained lower than predicted [2]. Meanwhile, generic tenofovir disoproxil (GxTD), a tenofovir prodrug, and emtricitabine (Gx-FTC) became available in August 2017. About $75 \%$ of all cART regimens worldwide were estimated to be based on tenofovir in 2015 [12] and it was found to be between $70 \%$ and $75 \%$ in the German market in 2017 [13]. Therefore, the availability of Gx-TD/FTC has the potential to induce significant dynamics in the ARV drug market [14], followed by substantial cost savings for the healthcare systems in both, developing [12,15] and industrialized $[16,17]$, countries. Interestingly, economists forecast price pressure to branded competitors, not just restricted to branded tenofovir disoproxil fumarate (TDF) but to the subsequently developed nucleotide analogue prodrug, tenofovir alafenamide (TAF), as well [18].

TAF became recently available as a component of cART and has shown similar efficacy but superior renal and bone safety compared with TDF in clinical studies [19-21]. In contrast to the analyses at week 48 and 96 [20,21], only the later update of 144 weeks data claimed superiority in the effectiveness of a specific TAF based fix combination co-formulated with elvitegravir/cobicistat/emtricitabine. But the inferior virological results at week 144 in the TDF arm were exclusively driven by missing values in the intention to treat Food and Drug Administration (FDA)-snapshot analysis, although the number of confirmed viral failures was slightly higher in the TAF group [22]. However, in November 2016, the German Federal Joint Committee (GBA) concluded that there is no additional benefit to the use of TAF/FTC versus TDF/FTC in ARV therapies [23,24]. Exclusively for its impact on pricing, the particular conclusion of the G-BA, an official authority by
German law is an important aspect in the design of the budget impact model described in this paper: The G-BA conclusion is rather thought as a hallmark for the German price negotiations between the social insurers and the manufacturer.

The aim of our model is to estimate the budget impact of generic TD/FTC over a 5 years' time horizon from the economic perspective of the payers of the German healthcare system for the tenofovir prodrug/ FTC containing double fix dose combination treatment segment. In order to ensure clarity, the analysis in our model is limited to the variation of few parameters, but it calculates the potential savings for two different scenarios. The underlying assumptions are discussed in detail against the background of existing scientific evidence on this topic.

\section{Methods}

A budget impact analysis (BIA) was performed to compare a hypothetical setting with and without the introduction of Gx-TD/ FTC for HIV treatment in the German healthcare system. The budget impact of treatment with Gx-TD/FTC was assessed every year up to 5 years, from the perspective of a healthcare payer (not including indirect costs such as productivity loss). Another objective of this analysis was to modulate maximum saving opportunity for the German healthcare system by using Gx-TD for the Tenofovir prodrug/Emtricitabine double combination market comprising branded TDF/FTC, Gx-TD/ FTC and TAF/FTC.

\section{Literature Search}

An explorative search using the Medline database conducted through December 2016 yielded 423 citations. Figure 1 shows the step-by-step disposition of the citation. The citations were screened by an experienced researcher for qualitative synthesis. Evidence was selected if it provided data related to either plausible model structure or appropriate input values related to the safety profiles of TAF and TDF in HIV. References within eligible papers were also scrutinized for related evidence using the snowball method $[25,26]$ and an additional hand search was applied. Non-English studies, non-human studies,

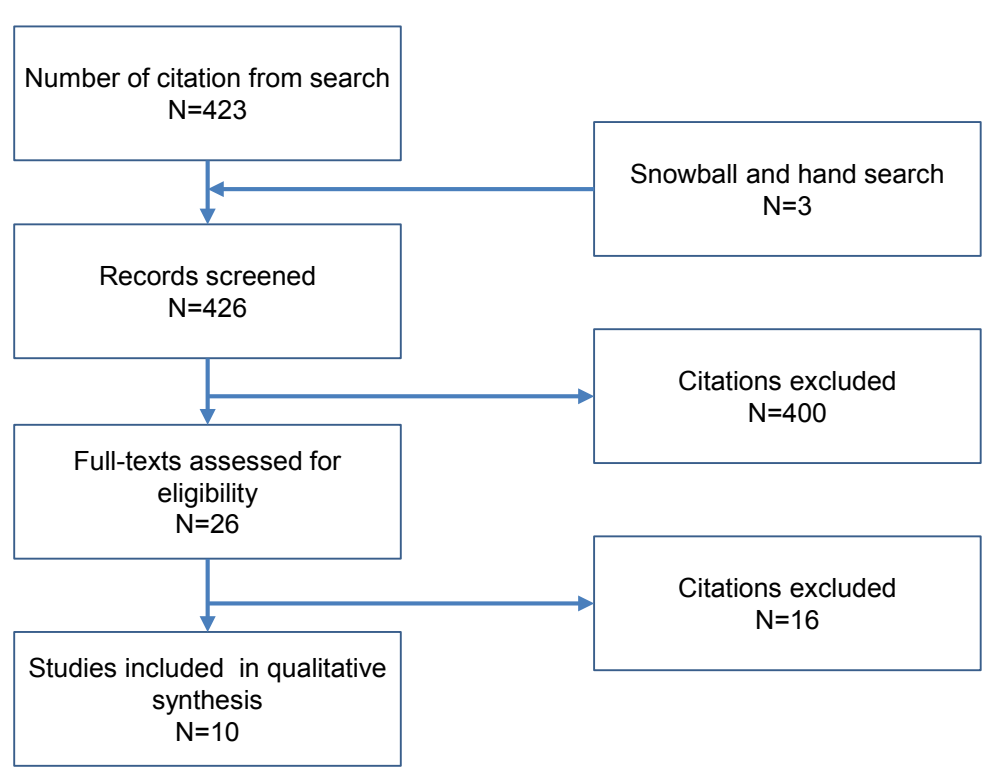

Figure 1: PRISMA literature search for model structure and safety profile. 
Citation: Stoll M, Balkin P, Goldbach J, Weber P, Peetroons B, et al. (2018) Budget Impact Analysis Highlighting the Cost-Saving Potential of Generic Tenofovir Disoproxil Based Antiretroviral Therapy in Comparison with Tenofovir Alafenamide Based Treatment Regimens in the German Healthcare System. J AIDS Clin Res 9: 754. doi: 10.4172/2155-6113.1000754

Page 3 of 9

case reports, notes and short surveys were excluded. Evidence based in European and US settings, focusing on the general population of HIV adults rather than subgroups, were selected. Subgroups such as children, patients who had received pre-exposure prophylaxis, pregnant women or patients with hepatitis B virus co-infection were excluded.

\section{Model Structure}

As shown in Figure 2, the model structure accounted for the number of diagnosed adult HIV patients (aged $\geq 18$ years) from the German healthcare system. This model focused exclusively on HIV patients who had been prescribed TDF/FTC or TAF/FTC as the nucleoside/nucleotide reverse transcriptase inhibitor (NRTI) backbone therapy. Backbone therapy is also given in fix combination with a third (triple therapy) or fourth agent (quadruple therapy), but our model focused solely on patients receiving the double combination plus separate $3^{\text {rd }}$ agent. No unbundling of single-tablet regimen (STR) containing 'TDF/FTC+X' was foreseen. The model did not cater to undiagnosed HIV patients, patients waiting for treatment or patients receiving alternative ARV treatments (other than combination therapy). After deriving a patient population, patients were divided between the comparator treatments (TDF/FTC or TAF/FTC) in two hypothetical worlds, with and without introduction of Gx-TD/FTC. In the first world, it was assumed that $\mathrm{Gx}$ TD/FTC was an option and thus Gx-TD/FTC can take patient share away from the comparator treatments (TDF/FTC or TAF/FTC). In the second world, it was assumed that in the absence of Gx-TD/FTC, patient share gets divided between currently available comparators. TDF/FTC and Gx-TD/FTC were assumed only to differ in terms of renal and bone safety as compared to TAF/FTC; therefore this BIA exclusively modelled renal and bone adverse events (AEs) [18]. Patients were subjected to treatment specific rates of AEs. Patients who experienced AEs (renal and bone) incurred costs associated with management of AEs as well as costs associated with switching to the next line of treatment. The BIA applied the differential costs for ARV treatment, monitoring costs and $\mathrm{AE}$ (renal and bone) management costs. Using this information, the model estimated the annual incremental costs and/or annual savings associated with introducing Gx-TD/FTC for a 5 year time horizon. The model was developed in MS Excel.

\section{Epidemiological Data}

Country specific epidemiological data for the modelling exercise were derived from the published literature. The numbers of treated HIV patients in Germany was 72,000 per Robert-Koch Institute, a German federal government agency and research institute and, one of the most important bodies for the safeguarding of public health in Germany. Baseline mean age of the HIV patient population was 43 years, with $17.9 \%$ of female patients [27]. Based on the recent data from Insight Health (July 2017), 36\% of treated HIV patients received either TDF/FTC or TAF/FTC as a double combination backbone therapy [28]. Therefore the number of patients entering our BIA was estimated as 25,920.

All-cause switching rate of patients from TDF/FTC and Gx-TD/ FTC to TAF/FTC was considered as $0.92 \%$, based on the study from Mocroft et al. [29]. This study investigated the potential increase in chronic kidney disease (CKD) associated with cumulative exposure to TDF and presented the incidence of CKD with cumulative exposure to TDF as ratios relative to non-exposure to TDF. The incidence of CKD was found to increase in the first 2 years of exposure to TDF, but it did not continue to increase significantly thereafter, which might suggest a threshold with respect to the drug's glomerular toxicity [29]. Inputs from this study were considered because the study included a European population and had an observational study design which was expected to have greater generalizability to real-world outcomes than randomized clinical trials. Mortality was excluded from the model as there were no differences in mortality between the comparators (TDF/ FTC vs. TAF/FTC) [30].

\section{Costs}

Three types of direct medical costs related to HIV treatment were

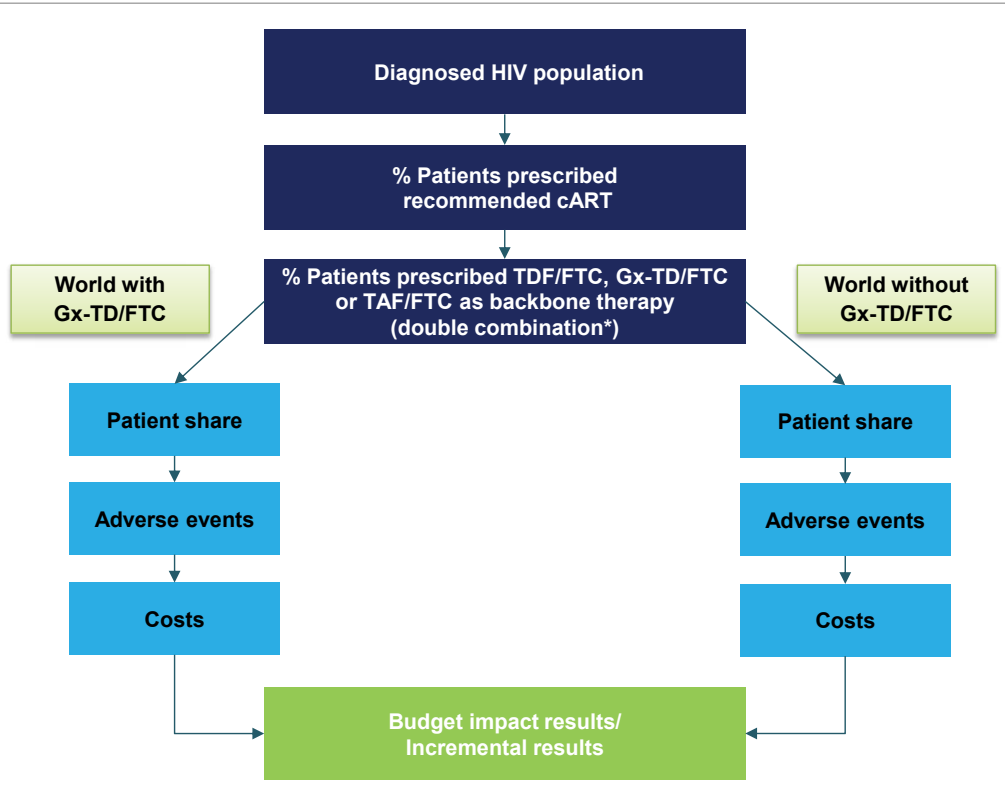

cART: Combination Anti-Retroviral Therapy; FTC: Emtricitabine; Gx-TD: Generic Tenofovir Disoproxil; HIV: Human Immunodeficiency Virus; TAF: Tenofovir Alafenamide; TDF: Tenofovir Disoproxil Fumarate

*No triple or quadruple therapies were included

Figure 2: Budget impact model flow. 
Citation: Stoll M, Balkin P, Goldbach J, Weber P, Peetroons B, et al. (2018) Budget Impact Analysis Highlighting the Cost-Saving Potential of Generic Tenofovir Disoproxil Based Antiretroviral Therapy in Comparison with Tenofovir Alafenamide Based Treatment Regimens in the German Healthcare System. J AIDS Clin Res 9: 754. doi: 10.4172/2155-6113.1000754

Page 4 of 9

included in this BIA: annual acquisition costs for ARVs, laboratory monitoring costs, and AEs (renal and bone) management costs. Official national list prices [31] and published label dose [32,33] were assumed to arrive at drug acquisition costs. For calculating laboratory monitoring costs and AE management costs, eligible data from the exploratory literature search were used. All costs were inflated to year 2016 using the consumer price index (CPI) for health [34]. Cost inclusion in the model followed International Society for Pharmacoeconomics and Outcomes Research (ISPOR) good research practice recommendations as published by Hay et al. [35]. Costs and health effects were discounted at the rate of $3 \%[36]$.

Patients incurred the cost of drug therapy as per their treatment arm. Prices of Gx-TD/FTC, TDF/FTC, and TAF/FTC were taken from the Lauer-Taxe price list [31]. For Gx-TD/FTC the Sandoz/Hexal product was used as reference. Prices were arrived by deducting the product specific rebates to statutory health insurance (SHI) (which is $6 \%$ manufacturer rebate for Gx-TD/FTC and TDF/FTC, $10 \%$ generic rebate for TDF/FTC and $0 \%$ for TAF/FTC), an additional rebate of $20 \%$ from the manufacturer's price was deducted from Gx-TD/FTC as the generic product is predominantly used in the context of SHI open house rebate contracts [37]. As a result, the prices per month were $€ 424$ for Gx-TD/FTC, €717 for TDF/FTC, and €655 for TAF/FTC. A calculation was also performed for a Gx-TD/FTC net price per month of $€ 100$. Laboratory monitoring costs were applied to patients taking either Gx-TD/FTC or TDF/FTC [38]. It consisted of annual monitoring of creatinine clearance and serum phosphate. Total monitoring cost was considered to be $€ 4.40$ in the first year and $€ 3.20$ in all the subsequent years $[39,40]$.

Upon experiencing an AE (renal and bone), patients incurred costs associated with the next line of treatment, which in both arms was TAF/FTC. Costs of managing AEs were assumed to be annual costs of CKD and costs associated with experiencing a fracture. These costs were assumed to be inclusive of all healthcare professionals' time and treatments. The mean annual direct medical cost per patient was assumed to be $€ 3,581$ in Germany for moderate and severe CKD $[41,42]$. The average one-off cost associated with bone fracture per patient was assumed to be $€ 9,962$ [43].

\section{Model Assumptions}

The BIA was built with certain key assumptions: Gx-TD/FTC was assumed to have similar antiviral efficacy as TDF/FTC. It was also assumed that TAF/FTC showed non-inferiority to TDF/FTC [19]. As per Mocroft et al. [29], all-cause switching rate of patients from TDF/FTC or Gx-TD/FTC to TAF/FTC was assumed to be $0.92 \%$ each year. Mortality was excluded from the model as there were no differences in the rate of mortality between the comparators [30]. The renal AE of interest were assumed to be $\mathrm{CKD}$, which the bone $\mathrm{AE}$ of interest was assumed to be a composite cost of fracture of the spine, arm, wrist and hip as used in the literature [44]. TAF/FTC was considered to be a treatment option with a superior safety profile compared with TDF/FTC or Gx-TD/FTC. TAF/ FTC patients were not at risk of renal AEs and experienced a baseline risk of fracture. TDF/FTC and Gx-TD/FTC patients were at risk of $\mathrm{CKD}$ and an increased risk of fracture. Annual laboratory monitoring costs for creatinine clearance and serum phosphate were applied to GxTD/FTC and TDF/FTC and not to TAF/FTC patients [45]. If a patient switched the treatment regimen owing to AEs, they continued to incur monitoring costs of their original treatment, as monitoring was assumed to be relevant to AEs.

\section{Scenario Analyses}

To test the impact of adoption of Gx-TD/FTC on budget, scenario analyses were conducted altering the patient share for Gx-TD/FTC. Two scenarios (realistic and maximum share) were considered keeping in mind the German healthcare system. It was assumed that Gx-TD/ FTC stabilises TAF/FTC market. Year-wise savings with Gx-TD/FTC were estimated to be between low value and high value throughout 5 years dependent on previously defined model assumptions.

In the realistic scenario, BIA calculated with net prices including SHI mandatory rebates. Patient share for Gx-TD/FTC reached $35 \%$ in the second year and remained at that rate until the fifth year by switching patients from TDF/FTC only. The maximum share scenario, i.e. the scenario that assumed the greatest patient share for Gx-TD/FTC, assumed that Gx-TD/FTC penetrates the German market aggressively. Previous patient shares (25\%) were used for the first year. To represent aggressive penetration, patient share for Gx-TD/FTC reached $80 \%$ by switching from TDF/FTC and TAF/FTC compared with 35\% in the realistic scenario. All other rebates remained similar as in the other scenario.

\section{Results}

Recent patient share evolvements of TDF/FTC and TAF/FTC before generic entry are shown in Figure 3. According to the PIA patient

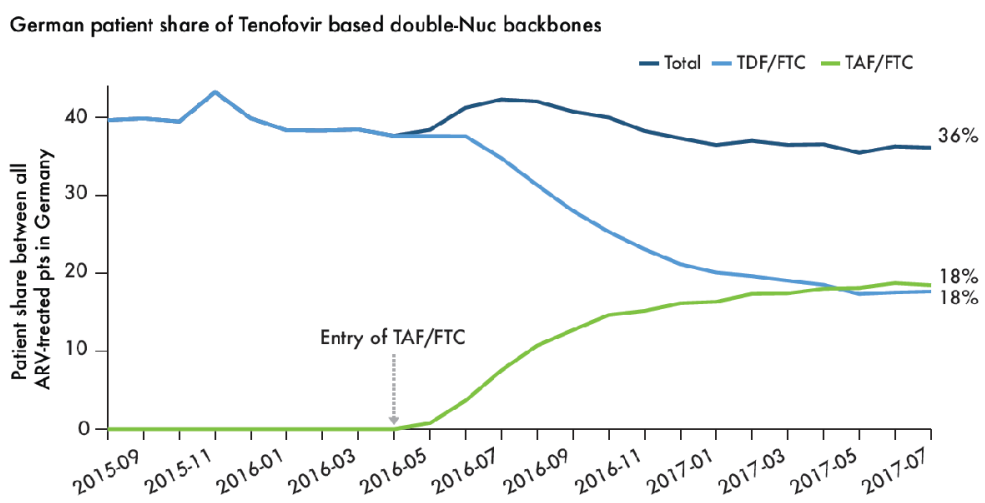

Source: PIA patient database from Insight Health (ATC-codes of analysed antiretroviral: ATC J05C1, J05C2, J05C3, J05C4, J05C5 and J05C9) ARV: Antiretroviral; FTC: Emtricitabine; pt: Patient; TAF: Tenofovir Alafenamide; TDF: Tenofovir Disoproxil Fumarate

Figure 3: German patient share of tenofovir based double combination backbone therapy (TDF/FTC or TAF/FTC). 
Citation: Stoll M, Balkin P, Goldbach J, Weber P, Peetroons B, et al. (2018) Budget Impact Analysis Highlighting the Cost-Saving Potential of Generic Tenofovir Disoproxil Based Antiretroviral Therapy in Comparison with Tenofovir Alafenamide Based Treatment Regimens in the German Healthcare System. J AIDS Clin Res 9: 754. doi: 10.4172/2155-6113.1000754

Page 5 of 9

database from Insight Health, the on-drug share of TDF/FTC and TAF/ FTC for all HIV treated patients was around 36\% (average of JanuaryJuly 2017) and had slightly decreased over the past year. This comprises $€ 212$ million treatment costs in Germany [13]. It is evident from the graph that since the launch of TAF/FTC, there has been a clear patient switch from TDF/FTC to TAF/FTC to approximately $50 \%$ (status July 2017) [28].

Savings generated by Gx-TD/FTC penetration were substantial in the BIA. Year-wise total expenditure with Gx-TD/FTC and without Gx-

\begin{tabular}{|l|c|c|c|c|c|c|c|c|c|c|}
\hline Setting & \multicolumn{4}{|c|}{ World without Gx-TD/FTC } & \multicolumn{5}{c|}{ World with Gx-TD/FTC } \\
\hline Year & $\mathbf{1}$ & $\mathbf{2}$ & $\mathbf{3}$ & $\mathbf{4}$ & $\mathbf{5}$ & $\mathbf{1}$ & $\mathbf{2}$ & $\mathbf{3}$ & $\mathbf{4}$ & $\mathbf{5}$ \\
\hline Gx-TD/FTC & 0 & 0 & 0 & 0 & 0 & 25 & 35 & 35 & 35 & 35 \\
\hline TDF/FTC & 50 & 45 & 40 & 35 & 30 & 25 & 10 & 10 & 10 & 10 \\
\hline TAF/FTC & 50 & 55 & 60 & 65 & 70 & 50 & 55 & 55 & 55 & 55 \\
\hline Total & 100 & 100 & 100 & 100 & 100 & 100 & 100 & 100 & 100 & 100 \\
\hline
\end{tabular}

FTC: Emtricitabine; Gx-TD: Generic Tenofovir Disoproxil; TAF: Tenofovir Alafenamide; TDF: Tenofovir Disoproxil Fumarate

All values in the table indicate percentages

Table 1: Patient share inputs in a world with and without GX-TD/FTC (realistic scenario).
TD/FTC in the realistic and maximum share scenarios are shown in Figures 4 and 5, respectively. These two graphs show a breakdown of costs in the two worlds specified: the world with Gx-TD/FTC and the world without Gx-TD/FTC, for each year of the 5 year time horizon. The total annual cost comprised costs associated with drug therapy, monitoring costs and AE management costs. The differences for each cost item between the two hypothetical worlds are presented (Tables 1 and 2).

The realistic scenario of Gx-TD/FTC use (growing to $35 \%$ by year 5)

\begin{tabular}{|l|c|c|c|c|c|c|c|c|c|c|}
\hline Setting & \multicolumn{4}{|c|}{ World without Gx-TD/FTC } & \multicolumn{5}{c|}{ World with Gx-TD/FTC } \\
\hline Year & $\mathbf{1}$ & $\mathbf{2}$ & $\mathbf{3}$ & $\mathbf{4}$ & $\mathbf{5}$ & $\mathbf{1}$ & $\mathbf{2}$ & $\mathbf{3}$ & $\mathbf{4}$ & $\mathbf{5}$ \\
\hline Gx-TD/FTC & 0 & 0 & 0 & 0 & 0 & 25 & 50 & 70 & 80 & 80 \\
\hline TDF/FTC & 50 & 45 & 40 & 35 & 30 & 25 & 10 & 10 & 10 & 10 \\
\hline TAF/FTC & 50 & 55 & 60 & 65 & 70 & 50 & 40 & 20 & 10 & 10 \\
\hline Total & 100 & 100 & 100 & 100 & 100 & 100 & 100 & 100 & 100 & 100 \\
\hline
\end{tabular}

FTC: Emtricitabine; Gx-TD: Generic Tenofovir Disoproxil; TAF: Tenofovir Alafenamide; TDF: Tenofovir Disoproxil Fumarate

All values in the table indicate percentages

Table 2: Patient share inputs in a world with and without Gx-TD/FTC (maximum patient share scenario).

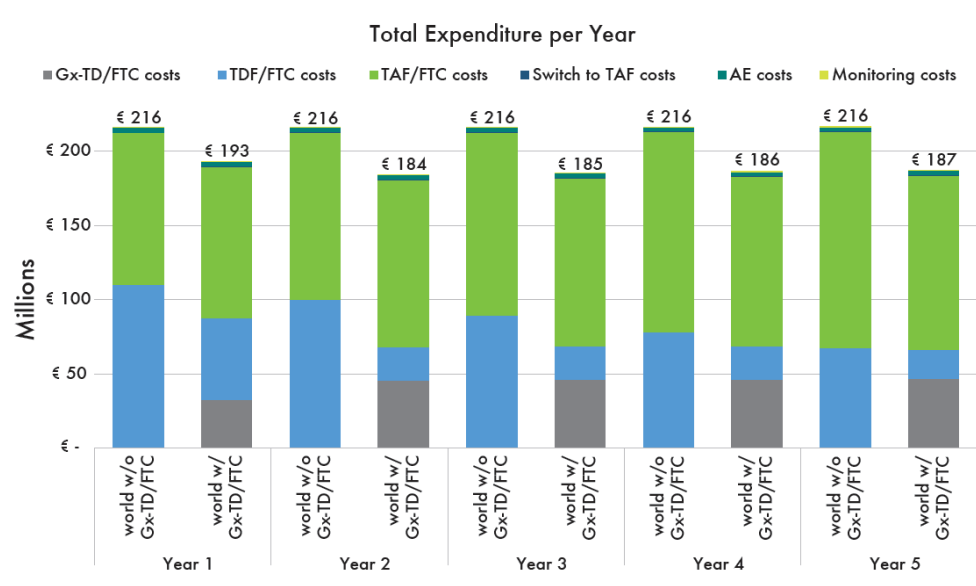

AE: Adverse event; FT: Emtricitabine; Gx-TD: Generic Tenofovir Disoproxil; TAF: Tenofovir Alafenamide; TDF: Tenofovir Disoproxil Fumarate; w/: With; w/o: Without

Figure 4: Year-wise total expenditure with and without Gx-TD/FTC (realistic scenario for the German healthcare system).

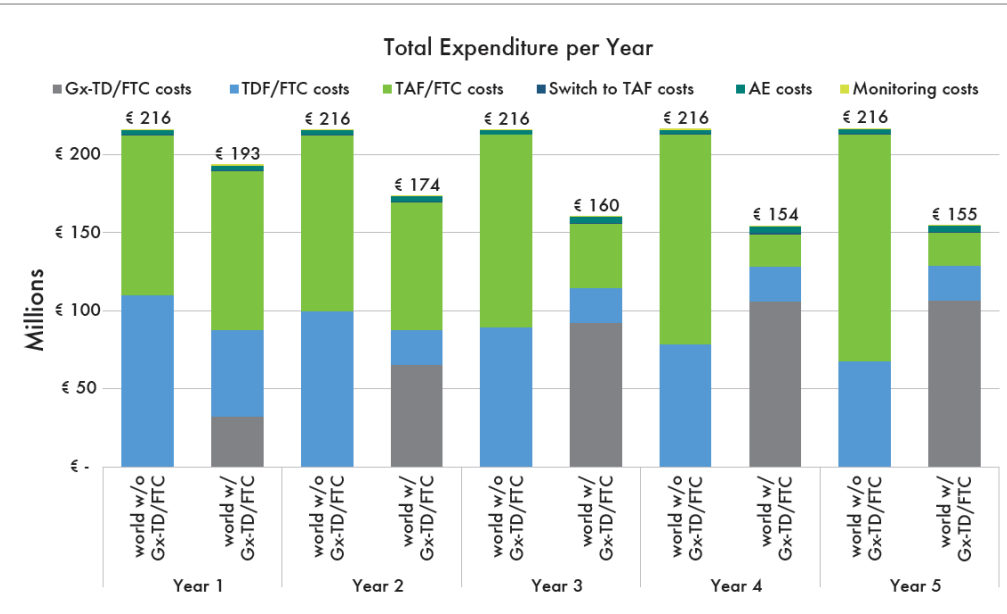

AE: Adverse event; FT: Emtricitabine; Gx-TD: Generic Tenofovir Disoproxil; TAF: Tenofovir Alafenamide; TDF: Tenofovir Disoproxil Fumarate; w/: With; w/o: Without Figure 5: Year-wise total expenditure with and without Gx-TD/FTC (maximum share scenario for the German healthcare system). 
Citation: Stoll M, Balkin P, Goldbach J, Weber P, Peetroons B, et al. (2018) Budget Impact Analysis Highlighting the Cost-Saving Potential of Generic Tenofovir Disoproxil Based Antiretroviral Therapy in Comparison with Tenofovir Alafenamide Based Treatment Regimens in the German Healthcare System. J AIDS Clin Res 9: 754. doi: 10.4172/2155-6113.1000754

Page 6 of 9

\begin{tabular}{|c|c|c|c|c|c|}
\hline Costs (in million $€$ ) & Year 1 & Year 2 & Year 3 & Year 4 & Year 5 \\
\hline \multicolumn{6}{|c|}{ Costs associated with patients who remain on initial treatment } \\
\hline Treatment costs without Gx-TD/FTC & 212.4 & 212.6 & 212.9 & 213.1 & 213.4 \\
\hline Treatment costs with Gx-TD/FTC & 189.8 & 180.8 & 181.8 & 182.8 & 183.7 \\
\hline Treatment drug costs, budget impact & -22.6 & -31.8 & -31.1 & -30.4 & -29.6 \\
\hline \multicolumn{6}{|c|}{ Costs associated with patients who switch to TAF/FTC } \\
\hline Switch to TAF/FTC costs without Gx-TD/FTC & 0.551 & 0.499 & 0.446 & 0.392 & 0.338 \\
\hline Switch to TAF/FTC costs with Gx-TD/FTC & 0.534 & 0.474 & 0.477 & 0.479 & 0.482 \\
\hline Switch to TAF/FTC drug costs, budget impact & -0.017 & -0.024 & 0.031 & 0.087 & 0.144 \\
\hline \multicolumn{6}{|l|}{ Laboratory monitoring costs } \\
\hline Monitoring costs without Gx-TD/FTC & 0.049 & 0.045 & 0.040 & 0.035 & 0.030 \\
\hline Monitoring costs with Gx-TD/FTC & 0.049 & 0.045 & 0.045 & 0.045 & 0.045 \\
\hline Monitoring costs, budget impact & 0 & 0 & 0.005 & 0.010 & 0.015 \\
\hline \multicolumn{6}{|l|}{ AEs management costs } \\
\hline AE costs without Gx-TD/FTC & 3.0 & 2.9 & 2.8 & 2.7 & 2.6 \\
\hline AE costs with Gx-TD/FTC & 3.0 & 2.9 & 2.9 & 2.9 & 2.9 \\
\hline AE management costs, budget impact & 0 & 0 & 0.1 & 0.2 & 0.3 \\
\hline \multicolumn{6}{|l|}{ Total costs } \\
\hline Total costs without Gx-TD/FTC & 215.9 & 216.0 & 216.2 & 216.3 & 216.4 \\
\hline Total costs with Gx-TD/FTC & 193.3 & 184.2 & 185.2 & 186.2 & 187.2 \\
\hline Budget Impact & -22.6 & -31.8 & -30.9 & -30.1 & -29.2 \\
\hline
\end{tabular}

AE: Adverse event; FTC: Emtricitabine; Gx-TD: Generic Tenofovir Disoproxil; TAF: Tenofovir Alafenamide; TDF: Tenofovir Disoproxil Fumarate Numbers may not add up due to application of rounding conventions

Table 3: Total costs with and without Gx-TD/FTC by year (realistic scenario)

\begin{tabular}{|c|c|c|c|c|c|}
\hline Costs (in million $€$ ) & Year 1 & Year 2 & Year 3 & Year 4 & Year 5 \\
\hline \multicolumn{6}{|c|}{ Costs associated with patients who remain on initial treatment } \\
\hline Treatment costs without Gx-TD/FTC & 212.4 & 212.6 & 212.9 & 213.1 & 213.4 \\
\hline Treatment costs with Gx-TD/FTC & 189.8 & 169.8 & 156.0 & 149.4 & 150.2 \\
\hline Treatment drug costs, budget impact & -22.6 & -42.8 & -56.9 & -63.8 & -63.2 \\
\hline \multicolumn{6}{|c|}{ Costs associated with patients who switch to TAF/FTC } \\
\hline Switch to TAF/FTC costs without Gx-TD/FTC & 0.551 & 0.499 & 0.446 & 0.392 & 0.338 \\
\hline Switch to TAF/FTC costs with Gx-TD/FTC & 0.534 & 0.630 & 0.842 & 0.952 & 0.957 \\
\hline Switch to TAF/FTC drug costs, budget impact & -0.017 & 0.131 & 0.396 & 0.560 & 0.619 \\
\hline \multicolumn{6}{|l|}{ Laboratory monitoring costs } \\
\hline Monitoring costs without Gx-TD/FTC & 0.049 & 0.045 & 0.040 & 0.035 & 0.030 \\
\hline Monitoring costs with Gx-TD/FTC & 0.049 & 0.059 & 0.080 & 0.090 & 0.091 \\
\hline Monitoring costs, budget impact & 0 & 0.015 & 0.040 & 0.055 & 0.060 \\
\hline \multicolumn{6}{|l|}{ AEs management costs } \\
\hline AE costs without Gx-TD/FTC & 3.0 & 2.9 & 2.8 & 2.7 & 2.6 \\
\hline AE costs with Gx-TD/FTC & 3.0 & 3.2 & 3.6 & 3.8 & 3.8 \\
\hline AE management costs, budget impact & 0 & 0.3 & 0.8 & 1.1 & 1.2 \\
\hline \multicolumn{6}{|l|}{ Total costs } \\
\hline Total costs without Gx-TD/FTC & 215.9 & 216.0 & 216.2 & 216.3 & 216.4 \\
\hline Total costs with Gx-TD/FTC & 193.3 & 173.7 & 160.4 & 154.2 & 155.0 \\
\hline Budget Impact & -22.6 & -42.4 & -55.7 & -62.1 & -61.4 \\
\hline
\end{tabular}

AE: Adverse event; FTC: Emtricitabine; Gx-TD: Generic Tenofovir Disoproxil; TAF: Tenofovir Alafenamide; TDF: Tenofovir Disoproxil Fumarate Numbers may not add up due to the application of rounding conventions

Table 4: Total costs with and without Gx-TD/FTC by year (maximum share scenario).

resulted in a saving of $€ 22.6$ million in the first year and in a maximum saving of $€ 31.9$ million ( $14.7 \%$ of total costs) in the second year as shown in Figure 4. Table 3 lists all the annual costs that were incurred by HIV patients in both arms (with and without Gx-TD/FTC).

In an alternative scenario associated with a maximum patient share (80\%) for Gx-TD/FTC, which includes switching from TDF/FTC and TAF/FTCs, savings increased to a maximum of $€ 62.1$ million $(28.7 \%)$ per year as shown in Figure 5. The savings reflected around 3\% to 6\% of the total German national costs for all ARVs [13].
Irrespective of the scenarios, annual drug treatment cost emerged as the largest contributor to the savings generated by introducing Gx-TD/ FTC in the German market. As shown in Tables 3 and 4, these annual savings ranged from $€ 22.6$ to $€ 31.8$ million in the realistic scenario and $€ 22.6$ to $€ 63.8$ million in the maximum share scenario. Costs associated with $\mathrm{AE}$ associated switching from Gx-TD/FTC to TAF/ FTC were also minimal by comparison. Under the realistic scenario, the annual cost of switching associated with AEs ranged from $€ 31,000$ to $€ 144,000$ from years 3 to 5 . The cost of switching associated with AEs per year was higher in the maximum share scenario than in the realistic 
Citation: Stoll M, Balkin P, Goldbach J, Weber P, Peetroons B, et al. (2018) Budget Impact Analysis Highlighting the Cost-Saving Potential of Generic Tenofovir Disoproxil Based Antiretroviral Therapy in Comparison with Tenofovir Alafenamide Based Treatment Regimens in the German Healthcare System. J AIDS Clin Res 9: 754. doi: 10.4172/2155-6113.1000754

scenario (€131,000 to €619,000). Annual costs of monitoring and AEs management were minuscule as compared to the drug treatment costs. Annual cost of monitoring in the realistic and maximum share scenarios ranged from $€ 0$ to $€ 15,000$ and $€ 0$ to $€ 61,000$, respectively. The maximum cost that was incurred per year in the management of AEs in the TDF/FTC or Gx-TD/FTC arm was $€ 300,000$ and $€ 800,000$ in the realistic and maximum share scenarios, respectively.

\section{Discussion}

The development and approval of innovative, efficacious and well-tolerated ARV drugs over the past few decades is an outstanding collaborative effort of basic, clinical and pharmaceutical research, study participants and social policy. An important incentive to carry out the highly expensive and economically risky research by pharmaceutical companies is the patent protection for successful approvals. The scope and duration of patent protection and the pricing of exclusive, essential medicines are subject to an evolving ethical discussion [46] and require a fair balance of interests between the involved parties, who must also recognize the access barriers associated with high costs of treatment [47]. This paves a critical role for generic drugs in any disease market.

The balance of interests in HIV infection as an infectious disease with variability in epidemiology also requires global political agreements. In an effort to match patient needs for ARV treatments with the demands of global drug development, drug pricing is country/ region specific. Higher drug prices often apply to rich countries where newer technologies are adopted more readily, whereas low-income countries have greater reliance on generics and their early availability [15]. While this convention holds true, high-income countries must also pay attention to the responsible use of economic resources of their healthcare systems. Part of this effort includes demanding early integration of generics into economically efficient treatment strategies [48]. Another approach to reduce costs is to accelerate the exploitation of potential savings by a rapid and comprehensive exchange on lowcost, substance-identical generic formulations [6].

Prescribing medicines in Germany is subject to the economic efficiency requirement enshrined in social legislation [49]. SHI may otherwise claim damage compensations directly from the prescribing physician. In this respect, the calculation of the aggressive scenario with a rather extensive exchange of branded tenofovir disoproxil fumarate against Gx-TD may, in future, be a realistic scenario for Germany. This would be in line with legislative initiatives to lower the price of prescription drugs [7] and would follow the existing criticism of moderate implementation of the legislation in Germany [6]. An aggressive exchange scenario has been calculated as an alternative in our BIA - it resulted in nearly doubling of the savings compared with a moderate scenario which is assumed in view of the existing conditions to be the rather realistic scenario.

Various arguments are made for carrying out a generic drug exchange within a functioning cART regimen on an individual basis only after weighing possible disadvantages. Owing to the special requirements for consistent compatibility and effectiveness of cART, a continuing controversy concerns the safety of a substance exchange even in the case of generics with identical active ingredients [50-52]. Some studies have described risks for sustainability of virological control by cART when the generic drug was associated with a higher number of pills [50,53]. Other studies found no difference in efficacy when switching to generic drugs [51] or considered concerns to be unfounded even against multiple exchanges, as long as the bioequivalence of the prescribed generic medicines was ensured [54].
However, in the field of ARV therapy, this concept has so far only been tested to a limited extent in clinical trials [55] or cohort studies [51] and therefore a more extensive integration of generic drugs into study concepts is demanded [14,48].

Interestingly, both patients and prescribers are open-minded and willing to share generic medicines [52]. Coincidentally, in two studies, about half of the patients, Engelhard et al. [56] and Krentz et al. [57] declared their consent to an exchange of elements of their cART even with a higher number of tablets, if the advantage would not only be theirs but a cost saving for their health insurance. The willingness was higher in older patients, after long-term therapy and higher educational or social status. Patients who were not in favor of exchange were more likely to have concerns about poorer efficacy, tolerability or convenience after an exchange of their existing cART regimen. In the realistic scenario in our BIA, we assume that patients and prescribers are not being driven by financial pressure or other repressions to change therapy, but by their own beliefs. In contrast to the studies by Engelhard et al. [56] and Krentz et al. [57], however, our BIA does not envisage an increase in the number of pills. Also, a switch from TAF/FTC to Gx-TD/FTC was excluded within this scenario. Therefore, it was assumed that the exchange rates used in the realistic scenario are a conservative estimate, which rather tends to underestimate the exchange rates in daily practice.

As described, our model assumes a superiority of TAF/FTC in terms of renal and bone safety but comparable effectiveness between TDF/FTC and TAF/FTC [19-21]. As this kind of TDF-associated clinically relevant AEs are usually not severe and, moreover, occur only rarely [29], they result in relatively low costs for the expense of the GxTD treatment group. For all cases with an AE, we calculated a switch to TAF-based cART with its resulting higher treatment and monitoring costs. In an effort not to underestimate any possible specific additional costs in the Gx-TD arm, we calculated for all cases changing from GxTD to TAF higher laboratory costs. This approach resulted in a slight overestimation of the costs for the Gx-TD arm, because in the German healthcare system, the reimbursement for laboratory tests is flat-rate based. Hence, the SHI would probably have no additional costs if all patients receive their comprehensive laboratory screening regularly in accordance with the treatment guidelines $[9,10]$. Furthermore, we did not calculate any potential cost impact of a less favourable lipid profile under TAF/FTC based cART compared with TDF/FTC. The clinical impact is not yet assessable, as only surrogate markers are available from clinical studies [22,58]. Considering that this would result in higher costs for lipid-lowering therapy and frequent cardiovascular events, these would not be reflected in the BIA. In this respect, there could be an underestimation of the costs incurred in favour of TAF-based ART, which would be in accordance with avoidance of any overestimation of TAF-related costs in the model.

Recently the majority of patients on cART in Germany receive a tenofovir based regime [13]. Hence it allows for the first time to estimate the potential of cost savings for the German SHI fund in a BIA based on real market prices since generic TD/FTC became available in Germany.

Interestingly the negotiated German price for the recently licensed TAF/FTC co-formulation was below the price for the branded TDF/ FTC co-formulated tablets from the same manufacturer. It confirms that price pressure by generics arises efficiently within the whole market of ARVs [18]. Our model was calculated with this actual price cut for TAF, and it revealed significant cost savings even then when Gx-TD/ FTC is used instead of branded TDF/FTC or TAF/FTC. Further price cuts for generic tenofovir are expected since Gx-TD/FTC is offered for use as a pre-exposure prophylaxis (PrEP) for a special price, which is 
Citation: Stoll M, Balkin P, Goldbach J, Weber P, Peetroons B, et al. (2018) Budget Impact Analysis Highlighting the Cost-Saving Potential of Generic Tenofovir Disoproxil Based Antiretroviral Therapy in Comparison with Tenofovir Alafenamide Based Treatment Regimens in the German Healthcare System. J AIDS Clin Res 9: 754. doi: 10.4172/2155-6113.1000754

Page 8 of 9

93\% below the price of branded TDF/FTC [59]. The realistic scenario employed in the BIA was conservative in nature, and savings would certainly be higher with increasing generic price rebates. This was tested by assuming a net drug price per month of $€ 100$ for Gx-TD/FTC. Under the patient share assumptions that was deemed most likely, this price of Gx-TD/FTC led to a saving of between $€ 47.6$ and $€ 67$ million (data not shown), with peak savings being achieved in the second year after GxTD/FTC implementation. Maximum patient share assumptions were also tested assuming a net drug price per month of $€ 100$ for Gx-TD/ FTC. Under these more generous assumptions, the introduction of $\mathrm{Gx}$ TD/FTC could result in a maximum saving of $€ 143.3$ million (data not shown), achieved in the fourth year after Gx-TD/FTC implementation. We were not able to implement the dynamics of generic price cuts and rebates e.g. arising by SHI tender contracts into our model because it would be a rather speculative approach. Since the cost savings are highly sensitive to the price of Gx-TD, higher savings would in any case result from the economic perspective of the German SHI fund: cost savings will be achieved with generic price reductions and branded ARV drug price reductions as well. The extent of savings in both cases depends on the availability of generic cART and the willingness to prescribe it extensively [18].

The limitations of the study result from its conception, as it focuses only on a few essential parameters and more differentiated aspects were not sufficiently represented. As a result, more far-reaching conclusions must be reserved for advanced analysis or future studies.

The strengths of the study are its limitation to a current market situation in the German healthcare system since the introduction of Gx-TD/FTC. The resulting clarity of the model and its limitation to two scenarios, one of which focused more on individualized treatment decisions and the other more on economically motivated restrictions of the treatment decision, allow a generally understandable representation of the model and its results. Furthermore, the chosen observation period was not so long that circumstances far in the future must appear as pure speculation. On the other hand, the period was long enough to give an imagination of a possible future shift in cost risks by choosing the therapy option with a potentially less favourable safety profile.

\section{Conclusion}

Savings for the German healthcare system with Gx-TD/FTC were substantial in the BIA.

- Maximum estimated savings per year: $15 \%$ ( $€ 31.9$ million) by the conservative, rather realistic approach with switches to $\mathrm{Gx}$ from TDF/FTC only.

- Maximum estimated savings up to $29 \%$ ( $€ 62.1$ million) per year of direct costs by a maximum share approach with switches to Gx from TDF/FTC and TAF/FTC.

- Savings reflect approximately $3 \%$ to $6 \%$ of the total German national costs for all ARVs.

- Savings would be higher with increasing generic price rebates or with resulting price cuts for branded comparators.

Our model makes cautious assumptions in favour of patient safety and care pathways.

- $\mathrm{CKD}$ (AE in this model) and associated costs would likely not be allowed to manifest in clinical practice since it would be monitored for within the conservative approach we made assumptions, which should exclude an overestimation of the savings in real clinical practice. Even after employing a conservative approach, this analysis highlights the cost-saving potential of Gx-TD/FTC for the German healthcare system.

\section{Acknowledgement}

We would like to thank Dr. Karin Grassmann and Dr. Carmen Bremer for their helpful and insightful comments.

\section{Declaration of Conflict of Interests}

Matthias Stoll received honoraria as an advisor, or a lecturer or funding for studies by the following pharmaceutical companies: Abbvie, Bristol-MyersSquibb Gilead, GlaxoSmithKline, Hexal, Hormosan, Janssen-Cilag, Merck, Sharp and Dohme, ViiV and ViroPharmaceuticals.

Phoebe Balkin is an employee of Novartis Business Services, Novartis Ireland Ltd., Ireland.

Joachim Goldbach received honoraria as a medical/scientific advisor by the following pharmaceutical companies: Wellcome, GlaxoWellcome, DuPont Pharmaceuticals, Bristol-Myers Squibb, Boehringer Ingelheim, Gilead, Celgene, Hexal and Sandoz.

Philipp Weber is an employee of Hexal AG, Industriestraße 25, 83607, Holzkirchen, Germany.

Baerbel Peetroons is an employee of Hexal AG, Industriestraße 25, 83607 , Holzkirchen, Germany.

Sameer Gokhale is an employee of Novartis Business Services, Novartis Healthcare Pvt. Ltd., India.

\section{References}

1. Antiretroviral Therapy Cohort C (2017) Survival of HIV-positive patients starting antiretroviral therapy between 1996 and 2013: A collaborative analysis of cohort studies. Lancet HIV 4: e349-e356.

2. Stoll M, Kollan C, Bergmann F, Bogner J, Faetkenheuer G, et al. (2011) Calculation of direct antiretroviral treatment costs and potential cost savings by using generics in the German HIV ClinSurv cohort. PLoS One 6: e23946.

3. Mostardt S, Hanhoff N, Wasem J, Goetzenich A, Schewe K, et al. (2013) Cost of HIV and determinants of health care costs in HIV-positive patients in Germany: Results of the DAGNÄ K3A study. Eur J Health Econ 14: 799-808.

4. Treskova M, Kuhlmann A, Bogner J, Hower M, Heiken H, et al. (2016) Analysis of contemporary HIVIAIDS health care costs in Germany: Driving factors and distribution across antiretroviral therapy lines. Medicine (Baltimore) 95: e3961.

5. Papot E, Landman R, Louni F, Charpentier C, Peytavin G, et al. (2017) Budget impact of antiretroviral therapy in a French clinic cohort. AIDS 31: 1271-1279.

6. Fischer KE, Stargardt T (2016) The diffusion of generics after patent expiry in Germany. Eur J Health Econ 17: 1027-1040.

7. Henschke C, Sundmacher L, Busse R (2013) Structural changes in the German pharmaceutical market: Price setting mechanisms based on the early benefit evaluation. Health Policy 109: 263-269.

8. lyengar S, Tay-Teo K, Vogler S, Beyer P, Wiktor S, et al. (2016) prices, costs and affordability of new medicines for hepatitis $C$ in 30 countries: An economic analysis. PLoS Med 13: e1002032.

9. http://www.daignet.de/site-content/hiv-therapie/leitlinien-1/Deutsch Osterreichische $\% 20$ Leitlinien $\% 20$ zur\%20antiretroviralen $\% 20$ Therapie $\% 20$ der\%20HIV_Infektion.pdf

10. http://www.eacsociety.org/guidelines/eacs-guidelines/eacs-guidelines.htm

11. https://aidsinfo.nih.gov/guidelines/html/1/adult-and-adolescent-arv/0

12. Gupta A, Juneja S, Vitoria M, Habiyambere V, Nguimfack BD, et al. (2016) Projected uptake of new antiretroviral (ARV) medicines in adults in low- and middle-income countries: A forecast analysis 2015-2025. PLoS One11: e0164619.

13. IMS Pharmascope (2017) Sell-Out in Units MAT 07 2017. Destatis, GraurheindorferStraße, Bonn, Germany.

14. Cattaneo D, Andreoni M, Carosi G, Cauda R, Lazzarin A, et al. (2017) Generic anti-retrovirals for the treatment of HIV: A novel challenge for Western countries? Int J Clin Pharmacol Ther 55: 381-393. 
Citation: Stoll M, Balkin P, Goldbach J, Weber P, Peetroons B, et al. (2018) Budget Impact Analysis Highlighting the Cost-Saving Potential of Generic Tenofovir Disoproxil Based Antiretroviral Therapy in Comparison with Tenofovir Alafenamide Based Treatment Regimens in the German Healthcare System. J AIDS Clin Res 9: 754. doi: 10.4172/2155-6113.1000754

Page 9 of 9

15. Amole CD, Middlecote C, Prabhu VR, Kumarasamy N (2017) Can the generic antiretroviral industry support access to a universal antiretroviral regimen? Curr Opin HIV AIDS 12: 390-397

16. Restelli U, Scolari F, Bonfanti P, Croce D, et al. (2015) New highly active antiretroviral drugs and generic drugs for the treatment of HIV infection: A budget impact analysis on the Italian National Health Service (Lombardy Region, Northern Italy). BMC Infect Dis 15: 323.

17. Hill A, Hill T, Jose S, Pozniak A (2014) Predicted savings to the UK National Health Service from switching to generic anti-retrovirals, 2014-2018. J Int AIDS Soc 17: 19497

18. Walensky RP, Horn TH, Paltiel AD (2016) The Epi-TAF for tenofovir disoproxil fumarate? Clin Infect Dis 62: 915-918.

19. Raffi F, Orkin C, Clarke A, Slama L, Gallant J, et al. (2017) Brief report: Longterm (96 week) efficacy and safety after switching from tenofovir disoproxil fumarate to tenofovir alafenamide in HIV-infected, virologically suppressed adults. J Acquir Immune Defic Syndr 75: 226-231.

20. Pozniak A, Arribas JR, Gathe J, Gupta SK, Post FA, et al. (2016) Switching to tenofovir alafenamide, coformulated with elvitegravir, cobicistat and emtricitabine, in HIV-infected patients with renal impairment: 48 week results from a single-arm, multicenter, open-label phase 3 study. J Acquir Immune Defic Syndr 71: 530-537.

21. Wohl D, Oka S, Clumeck N, Clarke A, Brinson C, et al. (2016) Brief report: A randomized, double-blind comparison of tenofovir alafenamide versus tenofovirdisoproxil fumarate, each co-formulated with elvitegravir, cobicistat and emtricitabine for initial HIV-1 treatment: Week 96 results. J Acquir Immune Defic Syndr 72: 58-64.

22. Arribas JR, Thompson M, Sax PE, Haas B, McDonald C, et al. (2017) Brie report: Randomized, double-blind comparison of tenofovir alafenamide (TAF) vs. tenofovir disoproxil fumarate (TDF), each coformulated with elvitegravir, cobicistat and emtricitabine (E/C/F) for initial HIV-1 treatment: Week 144 results. J Acquir Immune Defic Syndr 75: 211-218.

23. https://www.g-ba.de/downloads/39-261-2747/2016-11-03_AM-RL-XII_ Emtricitabin_Tenofoviralafenamid_D-228_BAnz.pdf

24. https://www.g-ba.de/downloads/40-268-4043/2016-11-03_AM-RL-XII Emtricitabin_Tenofoviralafenamid_D-228_TrG.pdf

25. Armstrong R, Jackson N, Doyle J, Waters E, Howes F (2005) It's in your hands: The value of hand searching in conducting systematic reviews of public health interventions. J Public Health (Oxf) 27: 388-391.

26. Jalali S, Wohlin C (2012) Systematic literature studies: Database searches vs. backward snowballing. Proceedings of the ACM-IEEE international symposium on Empirical software engineering and measurement.

27. https://www.rki.de/DE/Content/Infekt/EpidBull/Archiv/2016/Ausgaben/45_16. pdf? blob=publicationFile

28. http://www.insight-health.de/en

29. Mocroft A, Kirk O, Reiss P, De Wit S, Sedlacek D, et al. (2010) Estimated glomerular filtration rate, chronic kidney disease and antiretroviral drug use in HIV-positive patients. AIDS 24: 1667-1678.

30. Mills A Arribas JR, Andrade-Villanueva J, DiPerri G, Van Lunzen J, et al. (2016) Switching from tenofovirdisoproxil fumarate to tenofoviralafenamide in antiretroviral regimens for virologically suppressed adults with HIV-1 infection: A randomised, active-controlled, multicentre, open-label, phase 3 , non-inferiority study. Lancet Infect Dis 16: 43-52.

31. https://www.cgm.com/lauer-fischer/index.de.jsp

32. http://www.ema.europa.eu/docs/en_GB/document_library/EPAR_-_Product_ Information/human/000594/WC500043718.pdf

33. http://www.ema.europa.eu/docs/en_GB/document_library/EPAR_-_Product_ Information/human/004094/WC500207650.pdf

34. http://stats.oecd.org/Index.aspx?DataSetCode=CPL\#

35. Hay JW, Smeeding J, Carroll NV, Drummond M, Garrison LP, et al. (2010) Good research practices for measuring drug costs in cost effectiveness analyses: Issues and recommendations, the ISPOR drug cost task force report--Part I. Value Health 13: 3-7.
36. http://www.eunethta.eu/sites/5026.fedimbo.belgium.be/files/news attachments/2015-04-29-eco-gl_final_version.pdf

37. https://www.iqvia.com/de-de/locations/germany

38. http://www.ema.europa.eu/docs/en_GB/document_library/EPAR_Assessment_Report_-_Variation/human/000594/WC500226363.pdf

39. http://www.kbv.de/html/13259.php?srt=relevance\&stp=fulltext\&q=Kreatinin\&s= Suchen

40. http://www.kbv.de/html/13259.php?srt=relevance\&stp=fulltext\&q=Phosphor\&s =Suchen

41. Baumeister SE, Boger CA, Kramer BK, Doring A, Eheberg D, et al. (2010) Effect of chronic kidney disease and comorbid conditions on health care costs: A 10 year observational study in a general population. Am J Nephrol 31: 222-229.

42. Turchetti G, Bellelli S, Amato M, Bianchi S, Conti P, et al. (2017) The social cost of chronic kidney disease in Italy. Eur J Health Econ 18: 847-858.

43. Haussler B, Gothe H, Gol D, Glaeske G, Pientka L, et al. (2007) Epidemiology, treatment and costs of osteoporosis in Germany--The BoneEVA study. Osteoporos Int 18: 77-84.

44. Borges ÁH, Hoy J, Florence E, Sedlacek D, et al. (2017) Anti-retrovirals, fractures and osteonecrosis in a large international HIV cohort. Clin Infect Dis 64: $1413-1421$.

45. http://www.ema.europa.eu/docs/en_GB/document_library/EPAR_Assessment_Report_-_Variation/human/000594/WC500226363.pd

46. Pascual F (2014) Intellectual property rights, market competition and access to affordable anti-retrovirals. Antivir Ther 19: 57-67.

47. Rago L, Sillo H, t Hoen E, Zweygarth M (2014) Regulatory framework for access to safe, effective quality medicines. Antivir Ther 19: 69-77.

48. Pei PP, Weinstein MC, Li XC, Hughes MD, Paltiel AD, et al. (2015) Prioritizing HIV comparative effectiveness trials based on value of information: Generic versus brand-name ART in the US. HIV Clin Trials 16: 207-218.

49. http://www.sozialgesetzbuch-sgb.de/sgbv/12.html

50. Young J, Smith C, Teira R, Reiss P, Jarrín Vera I, et al. (2018) Antiretroviral pil count and clinical outcomes in treatment-naïve patients with HIV infection. HIV Med 19: 132-142.

51. Gianotti N, Poli A, Galli L, Franzin M, Tadini P, et al. (2017) Efficacy and safety of switching from branded to generic anti-retrovirals in virologically suppressed HIV-infected patients. PLoS One 12: e0182007.

52. Kieran JA, O'Reilly E, O'Dea S, Bergin C, O'Leary A (2017) Generic substitution of anti-retrovirals: patients' and health care providers' opinions. Int J STD AIDS 28: $1239-1246$.

53. Sweet DE, Altice FL, Cohen CJ, Vandewalle B (2016) Cost-effectiveness of single- versus generic multiple-tablet regimens for treatment of HIV-1 infection in the United States. PLoS One 11: e0147821.

54. Gwaza L, Gordon J, Welink J, Potthast H, et al. (2017) Interchangeability between first-line generic antiretroviral products prequalified by WHO using adjusted indirect comparisons. Antivir Ther 22: 135-144.

55. Galindo J, Amariles P, Mueses-Marín HF, Hincapié JA, et al. (2016) Effectiveness and safety of generic version of abacavir/lamivudine and efavirenz in treatment naïve HIV-infected patients: A non-randomized, open-label, phase IV study in Cali-Colombia, 2011-2012. BMC Infect Dis 16: 532

56. Engelhard EA, Smit C, Vervoort SC, Smit PJ, Nieuwkerk PT, et al. (2016) Patients' willingness to take multiple-tablet antiretroviral therapy regimens for treatment of HIV. Drugs Real World Outcomes 3: 223-230.

57. Krentz H, Campbell S, Gill V, Gill MJ (2017) Cost savings, patient perspectives on de-simplifying their single tablet co-formulated (STF) antiretroviral therapy for societal cost savings. HIV Med.

58. Gotham D, Hill A, Pozniak AL (2017) Candidates for inclusion in a universa antiretroviral regimen: tenofovir alafenamide. Curr Opin HIV AIDS12: 324-333.

59. Blasse C, Saalfeld S, Etournay R, Sagner A, et al. (2017) PreMosa: Extracting 2D surfaces from 3D microscopy mosaics. Bioinformatics 33: 2563-2569. 